\title{
Investigation on the nonlinear effect of a Gb/s visible light communication system utilizing DFT-S OFDM modulation
}

\author{
Can Wang ${ }^{1, a}$, Meng Shi ${ }^{2, b}$, Yiguang Wang ${ }^{3, c}$ and Nan Chi ${ }^{4, d}$ \\ 1,2,3,4 Department of Communication of science and engineering, Fudan University, Shanghai, \\ 200433, China \\ a13307130503@fudan.edu.cn, b13307130529@fudan.edu.cn, 'c12110720015@fudan.edu.cn, \\ dnanchi@fudan.edu.cn
}

Keywords: subcarrier numbers, dft-s ofdm.

\begin{abstract}
.
Visible light communication is a hot topic of wireless communication area which has drawn more attention to be researched. Dft-s ofdm is a main modulation method used in long term evolution (lte) in wireless communication, which is able to reduce the nonlinear limitation in client amplifier. Modulating directly by transmitter led in vlc will cause a strong linear effect which is harmful. As a result, this paper is aimed to do a research to study the anti-linear ability of $d f t-s o f d m$. The study shows that $d f t-s$ ofdm is helpful in reducing linear effect and papr. It also shows the simulation and experiment results to make sure the system can work safely and precisely in a certain situation. We make a conclusion that less number of subcarriers can help to lower papr and bit error ratio (ber).
\end{abstract}

\section{Introduction}

With the development of wireless communication technology, visible light communication which can improve performance in speed and other aspects catches people's eyes and become popular in communication area. Vlc is effective and feasible so that lots of researchers are busy doing experiments with the purpose of improvement of higher speed and lower bit error ratio. Such as cap, ofdm, bit loading ofdm, these are all applied in higher data transmission rate. Ofdm becomes an attractive modulation format for optical communications recently for its high spectrum efficiency, flexible coding scheme and obvious tolerance to intersymbol interference. But it is limited by papr. By our research, it suggested that papr can be controlled by some factors and several approaches can be adopted to reduce papr. As far as we know, there are measures such as clipping, pts, slm, coding, companding, active constellation, tone reservation which can reduce papr in wireless communication system. Lots of researchers considered a large quantities of methods to find out the relationship between these factors and reduction of papr. Few of these experiments pay more attention to the effect of subcarrier number. This paper provides a special view to show it. Recently, there is a method of using $d f t$ to finish frequency domain signal extensions to reduce papr. It has a nice characteristics on papr reduction which is similar to single-carrier signal's feature. What's more, simple algorithm is a highlight for the uplink requirements. This technique is named dft-s ofdm.

The paper is organized as follows. In the next section, main ideas and theories are given. Then, the matlab simulation for different numbers of subcarrier based on interleaving is presented. After that, next part presents the setup and experiment result. The experiment is aim to illustrate the efficiency of the different number of subcarriers. Finally, we conclude our paper.

\section{Theory and Discussions}

Before we learn about the detail of $d f t$-s ofdm, papr and $c c d f$ are presented as followed. The ofdm signal is composed of multiple independent subcarrier signal. This is different with the single carrier communication system. When the signal of subcarrier are in the same phase addition, the obtained synthetic signal may bring a high papr. We need a method named ccdf to measure the papr distribution. The complementary cumulative probability distribution function (ccdf) curve shows 
how much time the signal spends at or above a given power level. The power level is expressed in $d b$ relative to the average power. The percentage of time the signal spends at or above each line defines the probability for that particular power level. A $c c d f$ curve is a plot of relative power levels versus probability.

Dft-s ofdm is made up of these procedures approximately. Firstly, the time domain signal is divided into k blocks. Then they are converted into the frequency domain signal by $d f t$ respectively. Secondly, signal sequences from each block $d f t$ transform is allocated to each subcarrier. In this paper, the allocated method is interleaved allocation distribution. Each in turn from the choice of a symbol $d f t$ output sequences with different block is mapped to a set of consecutive subcarriers, until the completion of the distribution. As far as we know, $d f t-s$ method is a linear process, as a result, the $d f t-s$ ofdm can reduce the papr effectively.

\section{Simulation Analysis}

Several simulations through matlab have been presented to investigate the relationship between subcarrier number and papr reduction under visible light in the ofdm system.

As shown in Fig. 1 for vlc system based on $d f t-s$ ofdm, we figures out that subcarrier number is related to papr reduction. This simulation is under the condition: data number is equal to 1000 , qam order is equal to 4 (64qam), set number is equal to $2, d f t-s$ method is interleaved allocation. As can be seen from the picture, when carrier number is equal to 512, its line of $c c d f$ is taller than any other line of different number of subcarrier. From what is shown in Fig. 1, we can safely draw a conclusion: Lower the number of subcarrier is, better the papr performance of system is.

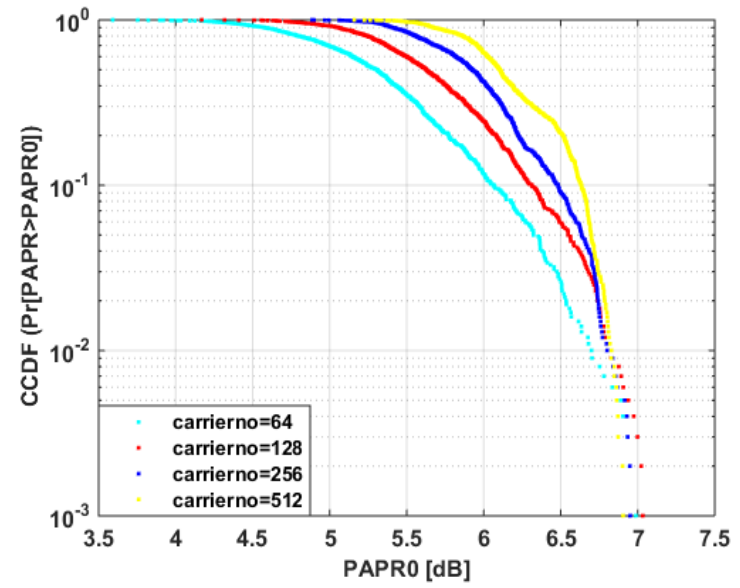

Fig. 1 papr performance of different subcarrier number in ideal vlc system.

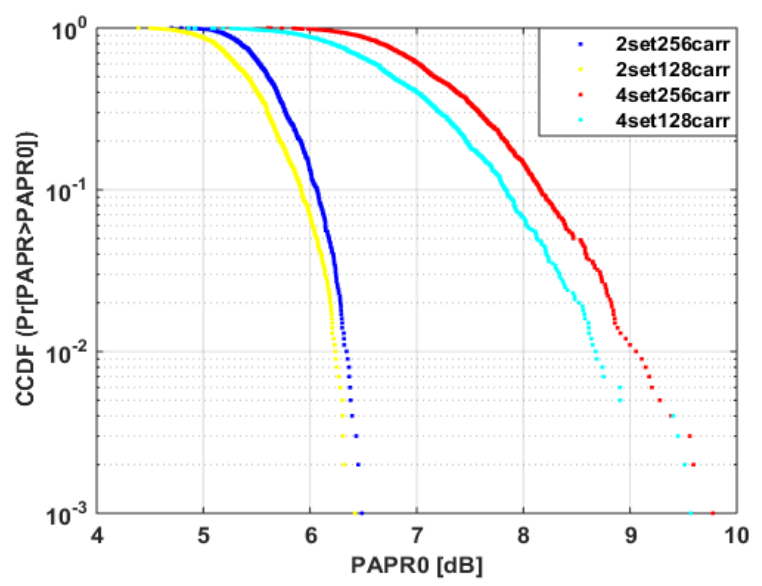

Fig. 2 papr performance of 128 and 256 subcarrier number in 2 and 4 sets

Furthermore, as shown in Fig. 2, this paper compares 128 and 256 carriers in 2 and 4 sets. From what is shown above, we can clearly find the subcarrier number play an important part in papr performance. The regulation is the same as of last simulation. Besides, performance of 2 sets is better than that of 4 sets. 


\section{Experimental Setup and Result}

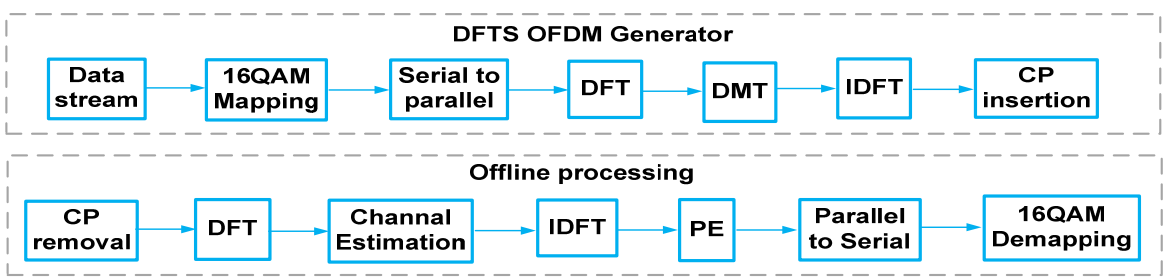

(a)

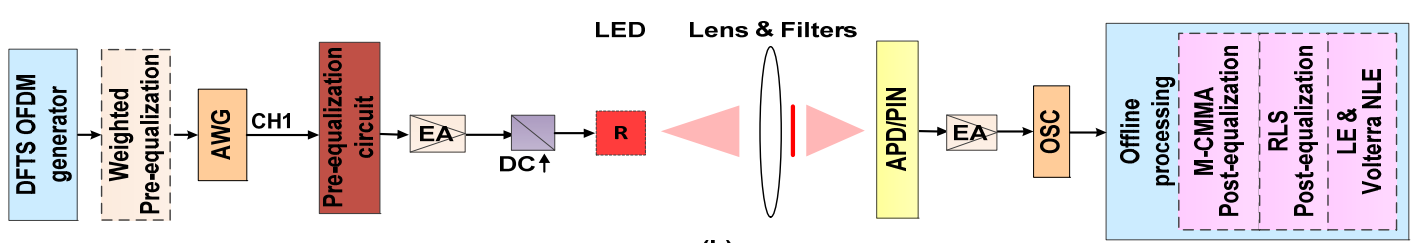

(b)

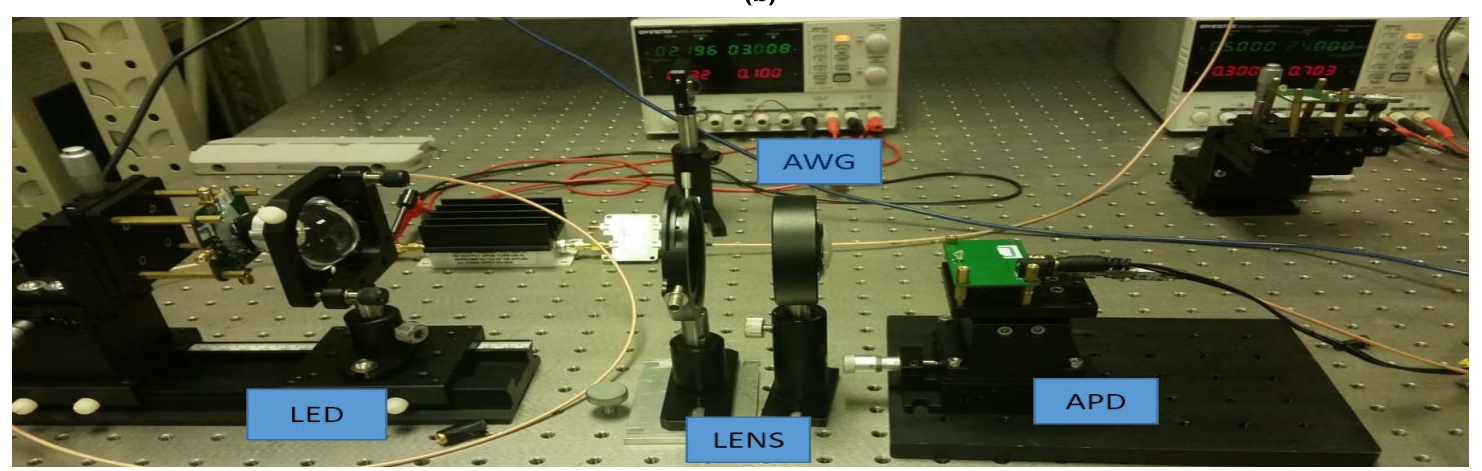

Fig. 3(a) (b) Experimental setup of DFT-S OFDM signal transmission

In order to illustrate the feasibility and effectiveness of different number of subcarriers, we construct a whole series of experimental facility. Fig. 3 indicates the experimental setup of $d f t-s$ ofdm signal transmission. The signal is generated off-line by matlab program and mapped to 64-qam constellation. The $d f t-s$ ofdm baseband signal is constructed with 128 or 256 subcarriers. When the subcarrier num -ber is equal to 128 , current is varied from $80 \mathrm{~mA}$ to $120 \mathrm{~mA}$, voltage is changed from $0.5 \mathrm{~V}$ to $1.2 \mathrm{~V}$. While subcarrier number is equal to 256 , the operation is the same. The ber is recorded to figure out the regulation. At the same time, we keep four constellation diagrams to find out whether 128 subcar -riers or 256 subcarriers is helpful to get a lower ber in transmission.

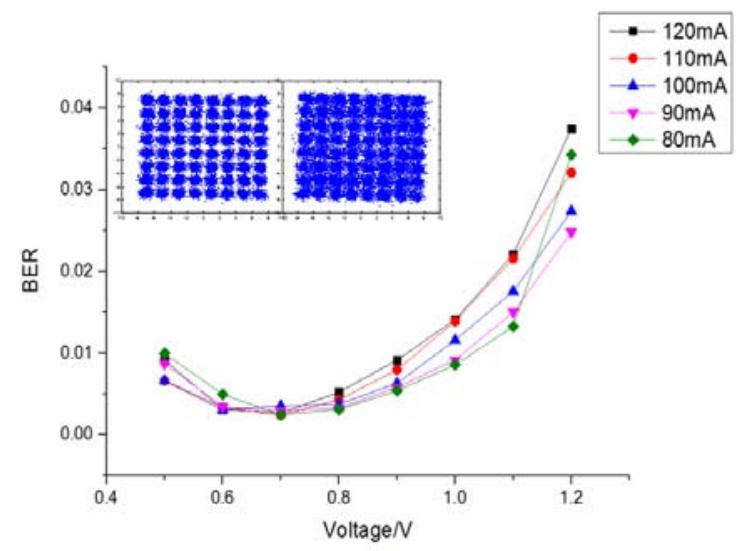

Fig. 4(a) 128 carriers and two constellation diagrams (best and worst)

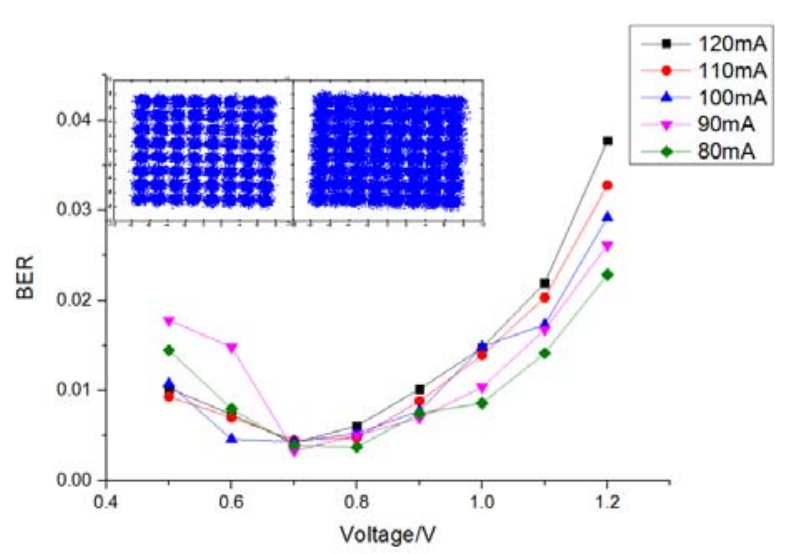

Fig. 4(b) 256 carriers and two constellation diagrams (best and worst)

From what we can see above, ber of 256 subcarriers is much bigger than that of 128 subcarriers. Also, comparing the constellation diagrams in those pictures is easy to show that subcarrier number is a significant factor to reduce papr in vlc system. In addition, when $r f$ voltage varies from about $0.7 \mathrm{~V}$ to $0.8 \mathrm{~V}$, the system is fit for all surrent. The bottom circle in the picture below is the best situation area. 


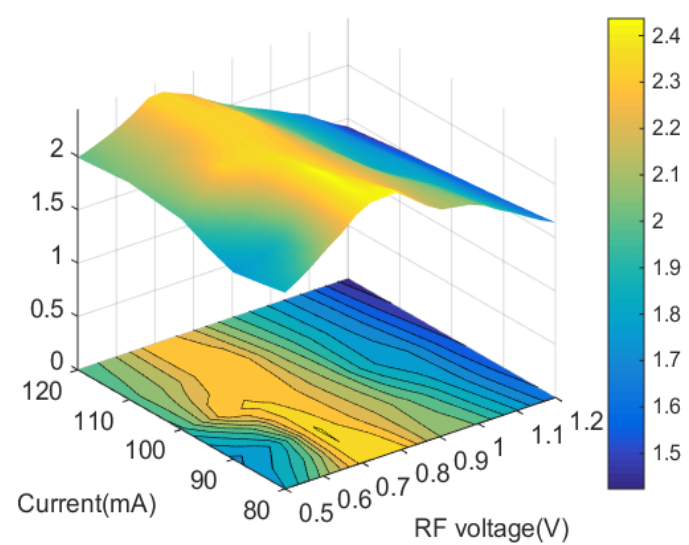

Fig. 4(c).The $3 d$ contour maps of $d f t-s$ ofdm (256 carriers and 128 carriers)

\section{Conclusions}

In this paper, for the first time, we explored $d f t-s$ ofdm modulation method in high-speed vlc system and achieve a gbit/s 64qam signal transmission using interleaving with different subcarriers in $d f t-s$ ofdm. The experimental results show that the bit error rate (ber) for an overall $1.2 \mathrm{gb} / \mathrm{s} 64 \mathrm{qam}$ signal over $40 \mathrm{~cm}$ transmission can be under the 7\% forward error correction (fec) limit of $3.8 \times 10-3$. The major advantage of this method is that it can solve high papr issues. Comparing a few of numbers of subcarriers of $d f t$-s ofdm, the smaller one has efficiency advantage to lower papr, reduce ber and imp -rove the performance. What's more, different number of sets, qam modulation order, real working condition, and other else all have multi effects on the system's performance. From all of our researc -hes, $d f t-s$ ofdm will be of great advantage in the high-speed vlc system. Further study will be focused on higher transmission speed, lower ber, and safety.

\section{Acknowledgements}

This work was financially supported by the national "863" program of china (no.2015AA016904) and the cast foundation project.

\section{References}

[1] Y. Fang, L. Tao and N. Chi, "Interleaved subcarrier allocation for DFT-spread OFDM to reduce PAPR", 17th Opto-Electronics and Communications Conference Technical Digest (OECC 2012), Busan, Korea, July 2012

[2] Nan Chi, Yiguang Wang, Yuanquan Wang, et al. Ultra-high-speed single red-green-blue light-emitting diode-based visible light communication system utilizing advanced modulation formats [J]. Chinese Optics Letters, 2014, 12(1): 010605.

[3] L. Tao, Jianjun Yu, Q. Yang, M. Luo, Z. He, Y. Shao, J. Zhang, N. Chi, "Spectrally efficient localized carrier distribution scheme for multiple-user DFT-S OFDM RoF PON wireless access systems", Optics Express, Vol. 20, No. 28, pp. 29665-29672 (2012).

[4] Li Tao, Ze Dong, Jianjun Yu, Nan Chi, Junwen Zhang, Xinying Li, Yufeng Shao, G. K. Chang, "Experimental demonstration of 48-Gb/s PDM-QPSK radio-over-fiber system over 40-GHz mm-wave MIMO wireless transmission”, [J] Photonics Technology Letters, 24(24), pp. 2276-2279 (2012). (SCI)

[5] J.Y. Shi, X.X. Huang, Y.G. Wang, L. Tao, N. Chi, "Improved Performance of a high speed $2 \times 2$ MIMO VLC network Based on EGCSTBC," ECOC, 2015 